Reseñas

\title{
La interculturalidad desde la perspectiva de la inclusión socioeducativa
}

Iván Manuel Sánchez Fontalvo ${ }^{1}$

${ }^{1}$ Universidad del Magdalena, Santa Marta, Colombia

Correspondencia: Iván Manuel Sánchez Fontalvo. Santa Marta: Universidad del Magdalena aaa@aaa.com

Por : Omar Huertas Díaz

El documento reseñado atiende a la preocupación de desarrollar una sociedad que sea capaz de responder a las necesidades actuales, que propenda hacia la equidad, inclusión e interculturalidad, al proponer espacios pedagógicos que permitan promover la formación de personas y colectivos con virtudes y capacidades para vivir en un contexto de pluralidad y diversidades humanas.

Considera el autor de forma acertada que desde la perspectiva de la interculturalidad, la inclusión y la educación, el desarrollo de competencias permite el respeto, el diálogo y la convivencia, lo cual termina por repercutir en el diario vivir y mejora la calidad de los pueblos.

Bajo este entendido, el documento se fundamenta en dos líneas de investigación, trabajadas por la Universidad del Magdalena, que responden a los planteamientos de a). Educación intercultural y b). Educación para el desarrollo de la ciudadanía y sociedad inclusiva, las cuales guían un trabajo por la educación para la diversidad, la inclusión y justicia social, formulando entonces, un modelo de educación que sea inseparable de la proyección política y social.

De esta forma analiza el autor la realidad sociocultural de Colombia, comprendiendo que se debe trabajar por una educación intercultural, desde la inclusión.

El libro se desarrolla a partir de tres capítulos fundamentales, el primero de ellos realiza un reconocimiento de la realidad sociocultural de Colombia, resalta la existencia de la diversidad étnica y cultural, contrastando estas características, con las esferas política, económica y social, que han generado violencia desde hace muchos años.

El capítulo segundo desarrolla el concepto de ciudadanía y su relación con la interculturalidad, visto desde la inclusión y la justicia social, se profundiza en la ciudadanía multicultural y ciudadanía intercultural.

Finaliza con el capítulo tercero donde se fundamenta el concepto de educación intercultural, partiendo de contextos multiculturales, se identifican las características de una educación intercultural.

El primer capítulo se denomina "La educación en contextos multiculturales", demuestra la gran diversidad de pueblos y culturas, y su preocupante situación de desigualdad económica, política y social, cuestionando el reconocimiento democrático de una constitución pluralista.

Se plantea la existencia en Colombia de 87 pueblos indígenas, con características económicas y sociales diversas, que conservan en su mayoría sus cosmovisiones, medicinas y regulaciones.

La población negra colombiana, denominada afrocolombiana, se aproxima a los 4.261.997 habitantes, distribuida principalmente en la Costa Caribe, Costa Pacífica, Región Andina, y en San Andrés, Providencia y Santa Catalina.

El concepto de afrocolombiano, se refiere a los niveles de mestizaje, al origen y no al color de la piel, nace como una necesidad de sus integrantes de obtener una identidad referencial, una resignificación de su humanidad y de sus raíces culturales.

Frente a la población gitana, o también llamada pueblo rom, se calcula que sus habitantes con ciudadanía colombiana ascienden a 4.832 
habitantes. Las principales se ubican en ciudades como Cúcuta, Girón, Bogotá, Cartagena, Cali y Barranquilla.

La población mestiza en Colombia, es aproximadamente un $60 \%$ y la blanca un $13 \%$. Se define como mestizo a las personas mezcla de europeos y comunidades indígenas; por su parte la población blanca son los descendientes de europeos que no tienen mezcla.

Una vez ubicadas las comunidades étnicas y culturales, pasa el autor a exponer las condiciones en las que se encuentran estas, esboza las condiciones de pobreza y desigualdad social, pese a su diversificada y enorme riqueza natural, pues el ingreso económico de una persona del grupo de los más ricos equivale a lo que reciben 60 personas del grupo de los más pobres, lo que refleja la inequidad presente, influye en esta estadística, la distribución de tierras, la poca prosperidad y la pobreza.

En dicho contexto es pertinente resaltar que las personas descendientes de africanos e indígenas, son invisibilizadas, se encuentran en situación de pobreza, desplazadas forzadamente, incluso mujeres cabeza de familia, todo ello sobre la razón de los estereotipos y prejuicios que conducen a la discriminación y segregación. Cuestiona entonces el autor el fenómeno del desplazamiento forzado como una gran tragedia humanitaria en Colombia, y la democracia colombiana.

Finaliza el capítulo describiendo la respuesta que la educación ha tenido para con este aterrador contexto, y obtiene como respuesta en este marco la educación popular y la etnoeducación.

Se entiende como educación popular, la práctica social que busca la transformación social, entendida desde dos posturas, la primera que la entiende como un proceso que debe realizarse fuera del estado, que prioriza la construcción de organización política revolucionaria, configurando procesos de referencia para crear un nuevo Estado; y la segunda entendiendo la educación popular como un proceso que debe hacerse con, y desde el Estado, pues se construye a través de ella una sociedad civil, capaz de ejercer en plenitud su ciudadanía, su propósito es el de restaurar una sociedad popular que dispute los intereses de los sectores dominantes presentes.

Por su parte, la etnoeducación es un proceso o estrategia útil en el propósito de reestructurar y desarrollar los pueblos, defender sus territorios y la supervivencia de sus identidades ancestrales. Según la ley general de la educación, la etnoeducación es una educación que se ofrece a grupos o comunidades que posean una cultura y fueros autóctonos, que debe estar ligada a los procesos sociales con el respeto de sus creencias y tradiciones.

En este marco de la etnoeducación se circunscribe la cátedra de estudios afrocolombianos, como una herramienta para la investigación, el análisis y la reflexión sobre la historia y problemáticas de poblaciones afrodescendientes.

Al respecto de estas formas de educación, el autor recalca las debilidades y carencias en la formación ciudadana, y pobreza de carácter material, pues sus instalaciones se encuentran en condiciones precarias y no responden a las necesidades y aspiraciones de grupos poblacionales.

El segundo capítulo llamado " El binomio interculturalidad e inclusión: Un debate académico en la configuración del concepto de ciudadanía", presenta de forma crítica la transformación del concepto de ciudadanía, pasando por el momento en el que la ciudadanía no responde a los fenómenos sociales, hasta el momento en que el concepto comienza a ajustarse a las problemáticas de la humanidad.

La ciudadanía tiene tres hitos históricos. El primero dado en la antigüedad clásica en las ciudades de Grecia y Roma, tomando la ciudadanía como un conjunto de obligaciones y derechos exclusivos de los hombres libres que pertenecían a una polis; el segundo hito, que puede llamarse la ciudadanía en el pensamiento liberal, en el cual la revolución francesa introduce el concepto de que el habitante de la ciudad o del Estado, es ciudadano, ya con el capitalismo industrial, se origina el sufragio de algunos ciudadanos considerados de primera, a lo cual responde Marx, reivindicando la igualdad de todos los ciudadanos por encima de sus condiciones económicas, culturales.

Y tercero, en el siglo $X X$, en el que el concepto de ciudadanía entra en crisis, desde su visión liberal, ya que a partir del llamado "estado bienestar" se busca una mediación social, y otorga una compensación de derechos a los más débiles.

Ahora, surgen conceptos nuevos de ciudadanía, 
incididos por los fenómenos sociales de injusticia y exclusión social, por motivos de intolerancia e irrespeto. Según estos fenómenos sociales, se originan diferentes conceptos de ciudadanía.

El primer fenómeno social que surge es el de la globalización, en el sentido de que se presentan unas dimensiones que hacen referencia a la generalización de ideas y valores globales, como pueden ser las categorías de derechos humanos, equidad de género, respeto a la diversidad étnica y cultural, esto genera el imaginario colectivo de un territorio que trasciende límites geográficos, y os hace objeto de derechos y obligaciones, lo cual origina el concepto de ciudadanía transnacional, que tiene como objetivo desarrollar el sentimiento de pertenencia a una comunidad de carácter global.

Se encuentra el fenómeno de la pluralidad y la multiculturalidad, refiriéndose al reconocimiento del valor de la diversidad, crea la necesidad de un concepto de ciudadanía no discriminatoria, da origen a una ciudadanía multicultural, basada en la solidaridad y el respeto; una ciudadanía intercultural, que tiene como ejes fundamentales el diálogo entre culturas, respeto por las diferencias y comprensión entre culturas; y una ciudadanía paritaria que supere los prejuicios.

Otro fenómeno de gran importancia es la exclusión, como resultado de la crisis del estado bienestar, que genera la ciudadanía social que busca luchar contra dicha exclusión, buscando la igualdad de oportunidades y equidad.

Se halla, de igual forma, el fenómeno de la apatía cívica, que se refiere al cuestionamiento de la democracia representativa, por la democracia participativa, lo que desarrolla la ciudadanía activa y responsable, integrada por la conciencia de pertenencia a una comunidad que implica compromiso cívico.

En este apartado propone el autor una ciudadanía intercultural e incluyente, como una iniciativa concretada entre un Estado que se caracteriza por su diversidad étnica y cultural, junto a los pueblos que la integran, buscando que todos comprendan, conozcan y compartan las diferentes culturas y problemáticas, tanto en el ámbito legal, político, como la reivindicación de sus derechos.

Se resalta como un aspecto relevante en la interculturalidad, el concepto de compartir, que supone el reconocimiento de las diferencias, como oportunidad para superar la exclusión, pues se permite desarrollar una actitud abierta y de aceptación de las diferencias, sin tratar de homogeneizarlas.

De igual forma el autor realiza una aproximación de estas nociones al contexto latinoamericano y la democracia, con lo que concluye el capítulo. Asevera el escritor que la interculturalidad permite construir un espacio para la interacción concertada entre diversos grupos, obteniendo como consecuencia un proceso de inclusividad, que busca la unidad a través de la diversidad.

Para terminar el libro, se desarrolla el tercer capítulo, titulado "La educación intercultural ante el reto de la inclusión"; en él se analiza la educación como protagonista y líder de la transformación social, desde la inclusión y por supuesto de la equidad.

Así, inicia el análisis describiendo la educación para la interculturalidad desde la perspectiva de la inclusión, en donde la educación para la ciudadanía es imprescindible pues se denota la permeabilidad de la escuela ante los cambios, y evolución de la sociedad.

Se reflexiona sobre la ciudadanía que requiere Colombia, caracterizada por ser democrática e intercultural, con capacidad de comprender cuatro modelos de ciudadanía: la multicultural, la intercultural, la democrática y la democrática radical.

Luego de esto, se da paso a la profundización de la educación para la interculturalidad, en el que el diálogo ente lo cultural y lo social es lo fundamental, lo cual significa que desde la educación se puede trabajar por el reconocimiento del derecho a la diferencia, y la eliminación de toda clase de discriminación y desigualdad social.

Se concibe la educación intercultural como la vía para el desarrollo de una sociedad más justa y equitativa, ya que desde allí se anima a la construcción de democracias verdaderas, donde el alumnado se involucre en la participación popular directa, lo cual permite dar posibles soluciones a los problemas de la institución y de la comunidad.

Por último se plantea la formación del profesorado en la educación intercultural desde la perspectiva de la inclusión, considerando que este ha de formarse en temas y contenidos que 
le permitan ahondar en la construcción de una sociedad intercultural, basados en principios como la justicia, equidad, diálogo y respeto activo, contextualizados a la comunidad en la que se presta el servicio.

La formación debe ser trabajada desde una orientación crítica y dialéctica con la realidad, que promueva el cambio del profesorado, empleando procesos innovadores y exitosos, desde las instituciones educativas, debido a que es allí donde se propician los espacios de investigación, reflexión y estudio, así que el momento apropiado para dicha preparación es el desarrollo del tiempo lectivo escolar.

Cierra el Doctor Iván Sánchez Fontalvo el libro reseñado, argumentando que la educación intercultural e inclusiva ha de desarrollarse en todos los centros educativos, buscando formar ciudadanos y ciudadanas que adquieran competencias para interactuar con distintos grupos, en el marco del respeto, diálogo, convivencia e intercambio de valores culturales, haciendo posible el impulso de una sociedad democrática y participativa. 